\title{
Anti-atherosclerotic effect of herbal extracts in N(G)-nitro-L-arginine methyl ester-treated rats
}

\author{
Senthil Nagarajan ${ }^{1} \cdot$ Rangachari Balamurugan $^{1} \cdot$ Eunju Shin $^{2} \cdot$ Kyu-Suk Shim $^{2}$ \\ - Min Jung Kim² Jeong Jun Lee ${ }^{3}$ Jae Kwon Lee ${ }^{1}$ (D)
}

Received: 22 July 2019 / Accepted: 19 August 2019 / Published Online: 30 September 2019

(C) The Korean Society for Applied Biological Chemistry 2019

\begin{abstract}
This study aimed to evaluate the anti-atherosclerotic and anti-hypertensive effects of six different plant extracts using a $\mathrm{N}(\mathrm{G})$-nitro-L-arginine-methyl ester (L-NAME)-induced rat model of hypertension. All extracts were administered orally for six weeks. At the end of the study period blood pressure, blood flow, aortic histopathology, and hepatic endothelial nitric oxide synthase (eNOS) expression were measured. Subsequently, we also measured the levels of intracellular reactive oxygen species, nitric oxide (NO), and anti-inflammatory cytokines in vitro. Based on these screening results, we selected extracts of Cinnamomum cassia $(C$. cassia) and Salvia miltiorrhiza (S. miltiorrhiza) for further evaluation. C. cassia and $S$. miltiorrhiza extracts ameliorated hypertension and atherosclerosis in L-NAME-treated rats in a dose-dependent manner. In addition, a mixture of C. cassia and S. miltiorrhiza had an additive effect to reduce blood pressure, increase blood flow, and normalize aortic tissue. This mixture demonstrated antioxidative and anti-inflammatory activities in vitro. In conclusion, although further analysis of the therapeutic mechanism is required, the anti-hypertensive and anti-atherosclerotic effects of this mixture are likely mediated by increased eNOS expression, and its anti-oxidative and anti-inflammatory activities.
\end{abstract}

Jae Kwon Lee $(\bowtie)$

E-mail: chemokine@cbnu.ac.kr

${ }^{1}$ Department of Biology Education, College of Education, Chungbuk National University, 1 Chungdae-ro, Seowon, Cheongju, Chungbuk, $362-$ 763, Republic of Korea

${ }^{2}$ Univera Co., Ltd., Seoul 04782, Republic of Korea

${ }^{3}$ R\&D center, Naturetech Co., Ltd., \#450-86, Maebong-Ro, Dongnam-Gu, Cheonan-Si, Chungnam 330-863, Republic of Korea

This is an Open Access article distributed under the terms of the Creative Commons Attribution Non-Commercial License (http://creativecommons. org/licenses/by-nc/3.0/) which permits unrestricted non-commercial use, distribution, and reproduction in any medium, provided the original work is properly cited.
Keywords Anti-oxidation · Atherosclerosis · Hypertension · Inflammation

\section{Introduction}

Hypertension has become one of the major causes of early death worldwide over recent decades. A recent World Health Organization report estimates there are one million people affected, and hypertension contributes to approximately $12.8 \%$ of annual mortality around the world [1]. Hypertension is diagnosed in more than $90-95 \%$ of these patients. In most cases, it is caused by endothelial defects involving nitric oxide (NO) depletion [2]. High blood pressure is one of the most important factors predisposing toward diseases, including heart failure, arthrosclerosis, myocardial infarction, and stoke. A lack of NO is a key defect in the development of hypertension and cardiovascular diseases [3]. Specifically, a reduction in NO synthesis contributes to the pathogenesis of high blood pressure, coronary artery disease, myocardial infarction, diabetes, and stroke. Treatment of rats or mice with N(G)-nitro-L-arginine-methyl ester (L-NAME) is a commonly used method for modeling high blood pressure in humans [4-6]. L-NAME-treated animals demonstrate reduced endothelial nitric oxide synthase (eNOS) expression in arteries, and a consequent reduction in plasma $\mathrm{NO}$, which results in narrower blood vessels and higher blood pressure. Thus, NO production is one of the most important factors in regulating cardiovascular function and homeostasis [5,7].

There are connections between the hypertension induced by LNAME and physiological stress markers. Specifically, a large dose of L-NAME $(60 \mathrm{mg} / \mathrm{kg} / 100 \mathrm{~mL}$ water/day) increases the levels of vascular superoxide, malondialdehyde, and plasma protein carbonyls [6,7]. These have been demonstrated to contribute to the etiology of hypertension in animals and humans, along with higher concentrations of reactive oxygen species (ROS) [7]. Oxidative stress mechanisms have been well documented by in vitro and in 
vivo studies of L-NAME-treated rats [8-12], involving the production of large quantities of ROS. Furthermore, damage to vascular tissue in L-NAME-treated rats results in high ROS concentrations and greater secretion of proinflammatory cytokines [8-12].

Asians frequently use herbal remedies as alternative medicines, because of their lower cost, perceived efficacy, and tolerability [13]. Several medicinal plant extracts have been used to prevent degenerative diseases. Cinnamomum cassia (C. cassia) and Salvia miltiorrhiza ( $S$. miltiorrhiza) are frequently used in Chinese traditional medicines and are routinely used to treat hypertensive diseases [14]. However, there have been no studies of using these herbs in combination. In the present study, we aimed to determine whether a combination of $C$. cassia and $S$. miltiorrhiza extracts can improve blood flow and vasodilation, and reduce inflammation, using in vitro and in vivo models.

\section{Materials and Methods}

\section{Plant extract preparation}

A series of candidate plants (C. cassia, Crataegus pinnatifida Bunge, Eucommia ulmoides, Morus nigra L. fruit, Prunella vulgaris, and S. miltiorrhiza) were purchased from Kyungdong oriental herb market in Korea and a medical herb market in China. The voucher specimens (EDU001-EDU019) are stored at Chungbuk National University, Korea. Plants were extracted with ethanol and/or water as reported by Yang et al. [15], and the content of marker compound in various extracts were determined by spectrometric method or HPLC method (data not shown).

C. cassia bark was extracted three times in $100 \%$ ethanol for $2 \mathrm{~h}$. The extract of the aerial parts was not less than 5\% polyphenol. After filtration, the extract was vacuum-concentrated to dryness. Crataegus pinnatifida (C. pinnatifida) Bunge fruit extract was extracted in $70 \%$ ethanol, contained $5 \%$ procyanidins, and concentrated to dryness using a spray dry system. Eucommia ulmoides (E. ulmoides) bark was extracted in $70 \%$ ethanol, contained $2.5 \%$ chlorogenic acid, and concentrated to dryness using a spray dry system. Morus nigra (M. nigra) L. fruit was extracted in $70 \%$ ethanol, contained $25 \%$ anthocyanins, and concentrated to dryness using a spray dry system. Prunella vulgaris ( $P$. vulgaris) was extracted in water three times for $2 \mathrm{~h}$, with the extract containing $2 \%$ flavone, and concentrated to dryness using a vacuum system. S. miltiorrhiza root was extracted in $70 \%$ ethanol, contained $9.5 \%$ salvianolic acid B, and concentrated to dryness using a spray dry system.

\section{Animals}

Healthy adult male Wistar rats were procured from the Dooyeol biotech Lab, South Korea, maintained at constant temperature $\left(23-25^{\circ} \mathrm{C}\right)$ and under a $12 \mathrm{~h}$ light $/ 12 \mathrm{~h}$ dark cycle, and fed a standard chow diet. After an acclimation period of seven days, the rats were allocated to specific treatment groups based on body weight, using a randomized block design. The animal study was approved by the Ethics Committee for Animal Experimentation of Chungbuk National University (Permit Number: CBNUR-909-15, Korea).

\section{Design of the in vivo screening study}

The rats were allocated to eight experimental groups. The normal control group received sterilized drinking water, and the same water was administered intragastrically for six consecutive weeks. The L-NAME hypertensive control group was administered LNAME (60 mg/kg/100 mL water/day) in sterilized drinking water, and the same water was administered intragastrically for six consecutive weeks. The treatment groups were administered LNAME in sterilized drinking water, and each plant extract (300 $\mathrm{mg} / \mathrm{kgbw}$ per day) was administered intragastrically for six consecutive weeks. No treatment-related mortality or morbidity was observed during the experimental period. The rats' body weights were measured on the initial and final days of the treatment period. On the day of sacrifice, blood flow rate was measured according to the manufacturer's instructions (Flo- $\mathrm{Cl}$; Omegawave, Inc., Tokyo, Japan).

\section{Measurement of blood pressure}

The rats' blood pressure was measured, noninvasively and without anesthesia, with an indirect tail cuff method (Harvard Apparatus, Millis, MA, USA). The rats were restrained on a warming plate and acclimated for $5 \mathrm{~min}$ before the cuff was fitted. Blood pressure was measured twice during the experimental period.

\section{Estimation of blood flow in the carotid artery}

The untreated control, L-NAME control, and extract-treated animals were anesthetized with urethane (Sigma-Aldrich, St. Louis, MO, USA); then the carotid artery was exposed. Blood flow was then measured using laser Doppler flowmetry (Flo-C1; Omegawave, Inc.) and analyzed using Chart 5 software (eDAQ Pty Ltd, NSW, Australia).

\section{Sampling}

After six weeks of treatment, the rats were anesthetized with urethane, and blood samples were collected from the caudal vena cava. The collected blood samples were centrifuged at 3,000 rpm for $15 \mathrm{~min}$ to separate sera for biochemical analyses. Afterwards, liver samples were collected and stored at 80 until analyzed.

\section{Histopathologic analysis}

After euthanasia, the ascending aorta was collected from each rat and fixed in $10 \%$ formaldehyde solution. The tissues were fixed, processed, and paraffin-embedded, then sectioned at $5 \mu \mathrm{m}$ thickness, stained with hematoxylin and eosin (H\&E), and examined using a light microscope [3]. Image analysis was performed using analySIS $^{\circledR}$ TS auto (Olympus Soft Imaging System, Münster, 
Germany) to quantify the aortic diameter and vessel wall thickness.

\section{Design of the confirmatory study}

Based on the screening study results, we selected two plant extracts (of C. cassia and S. miltiorrhiza) for further study. Rats were allocated to seven groups of 10 rats each. Hypertension was induced using L-NAME, as described above, and the rats were treated once daily for six weeks with extracts of C. cassia (150 $\mathrm{mg} / \mathrm{kgbw}$ per day), S. miltiorrhiza $(150 \mathrm{mg} / \mathrm{kgbw}$ per day), or mixtures of $C$. cassia (50\%) and $S$. miltiorrhiza $(50 \%)(150,300$, or $600 \mathrm{mg} / \mathrm{kgbw}$ per day). On the day of sacrifice, blood pressure and blood flow rate were measured according to the manufacturers' instructions.

\section{Western blot analysis}

Total NOS activity was determined in crude liver homogenates using a commercial kit. Briefly, equal amounts of protein were separated using sodium dodecyl sulfate-polyacrylamide gel electrophoresis and transferred to polyvinylidene difluoride membranes. The membranes were blocked with blocking buffer for $1 \mathrm{~h}$ at $4{ }^{\circ} \mathrm{C}$ and then incubated with anti-eNOS antibody (Abcam, Cambridge, UK) overnight at $4{ }^{\circ} \mathrm{C}$. They were then washed four times for $10 \mathrm{~min}$ in TBST buffer, incubated with secondary antibody for $2 \mathrm{~h}$ at room temperature, and then washed again four times for $10 \mathrm{~min}$ in TBST. eNOS expression was normalized to that of $\beta$-actin, using the antibody supplied by the manufacturer. Bound antibodies were identified by chemiluminescence.

\section{Nitric oxide release assay}

HUVEC cells were seeded in 96-well plates at $3 \times 10^{4}$ cells/well and incubated for $24 \mathrm{~h}$. The medium was then removed, the cells were pretreated for $30 \mathrm{~min}$, and then they were incubated with 500 $\mu \mathrm{M} \mathrm{H}_{2} \mathrm{O}_{2}$ for $4 \mathrm{~h}$. The total NO content was then measured in the test medium using an NO assay kit (R\&D systems, MN, USA).

\section{Intracellular ROS production}

HUVEC cells were seeded in 96-well black plates at $3 \times 10^{4}$ cells/ well and incubated for $24 \mathrm{~h}$. After the medium was removed, the cells were washed once with assay buffer, treated with $25 \mu \mathrm{M}$ of $2^{\prime}, 7^{\prime}$-dichlorofluorescein, and then incubated for $45 \mathrm{~min}$ in a $37^{\circ} \mathrm{C}$ incubator. After the reaction, the sample was washed once with assay buffer and pretreated for $30 \mathrm{~min}$; then, after the addition of $500 \mu \mathrm{M} \mathrm{H}_{2} \mathrm{O}_{2}$ for $1 \mathrm{~h}$, absorption was measured using excitation and emission wavelengths of $485 / 530 \mathrm{~nm}$.

\section{Enzyme-linked immunosorbent assay (ELISA)}

RAW264.7 cells were seeded in 96-well plates at $1 \times 10^{5}$ cells/well and incubated for $24 \mathrm{~h}$. After removal of the medium and pretreatment for $1 \mathrm{~h}$, lipopolysaccharide $(1 \mu \mathrm{g} / \mathrm{mL})$ was added for $24 \mathrm{~h}$. Pro-inflammatory cytokine concentrations were then measured in the media using ELISA assays (R\&D systems) per the manufacturer's instructions.

\section{Statistics}

Statistical analyzes of the data were performed using Student's $t$ test. Values are stated as the mean $\pm \mathrm{SD}$.

\section{Results}

Body weight during the in vivo screening study

To confirm the extracts' safety, we monitored the rats' body weight, mortality, and clinical signs. All of the rats survived until their scheduled necropsy, and no gross abnormalities were observed during the study that could be related to the plant extracts. The mean body weights of the rats were recorded on day 0 and, as shown in Fig. 1A, the rats were assigned to groups to minimize body weight differences. At the end of the experiment, there were no statistically significant differences in body weight among the experimental groups.

\section{Blood flow in the carotid artery}

L-NAME treatment is known to induce hypertension and harden arteries, which reduces the blood flow. As expected, blood flow was reduced by L-NAME treatment (Fig. 1B). However, there were increases in blood flow after administration of the crude plant extracts prepared from C. cassia, C. pinnatifida, E. ulmoides, $M$. nigra, $P$. vulgaris, and $S$. miltiorrhiza for six weeks. Of these six extracts, treatment with the $C$. cassia extract had the largest effect on blood flow, near-normalizing it. Although its effect was variable, S. miltiorrhiza extract had the second-largest effect.

\section{Effect of $C$. cassia and $S$. miltiorrhiza extracts on rat body weight in the confirmatory study}

Based on the results of the screening studies, we selected two plant extracts (of C. cassia and S. miltiorrhiza) for further study. In the preliminary experiment, we observed no gross abnormalities or mortality after treatment with $300 \mathrm{mg} / \mathrm{kgbw}$ of the extracts (Fig. 1). Therefore, we increased the dose to a maximum of 600 $\mathrm{mg} / \mathrm{kgbw}$ for the follow-up experiment, but even at the highest dose, no gross abnormalities or mortality were observed during the study period. The rats' mean body weights on the day of sacrifice were similar among the groups (Fig. 2A).

\section{Effect of $C$. cassia and $S$. miltiorrhiza extracts on blood pressure}

Oral administration of L-NAME induced significant increases in systolic blood pressure (SBP), diastolic blood pressure (DBP), and mean arterial pressure (MAP) compared to the normotensive control rats (Table $1 ; 44,51$, and $41 \%$, respectively). High blood pressures were reduced by treatment with $C$. cassia and $S$. miltiorrhiza extracts, either singly or in combination. Moreover, the combination extracts administered at $600 \mathrm{mg} / \mathrm{kgbw}$ reduced these parameters in a dose-dependent manner (by 24, 30, and $26 \%$, respectively) compared to the L-NAME group. 

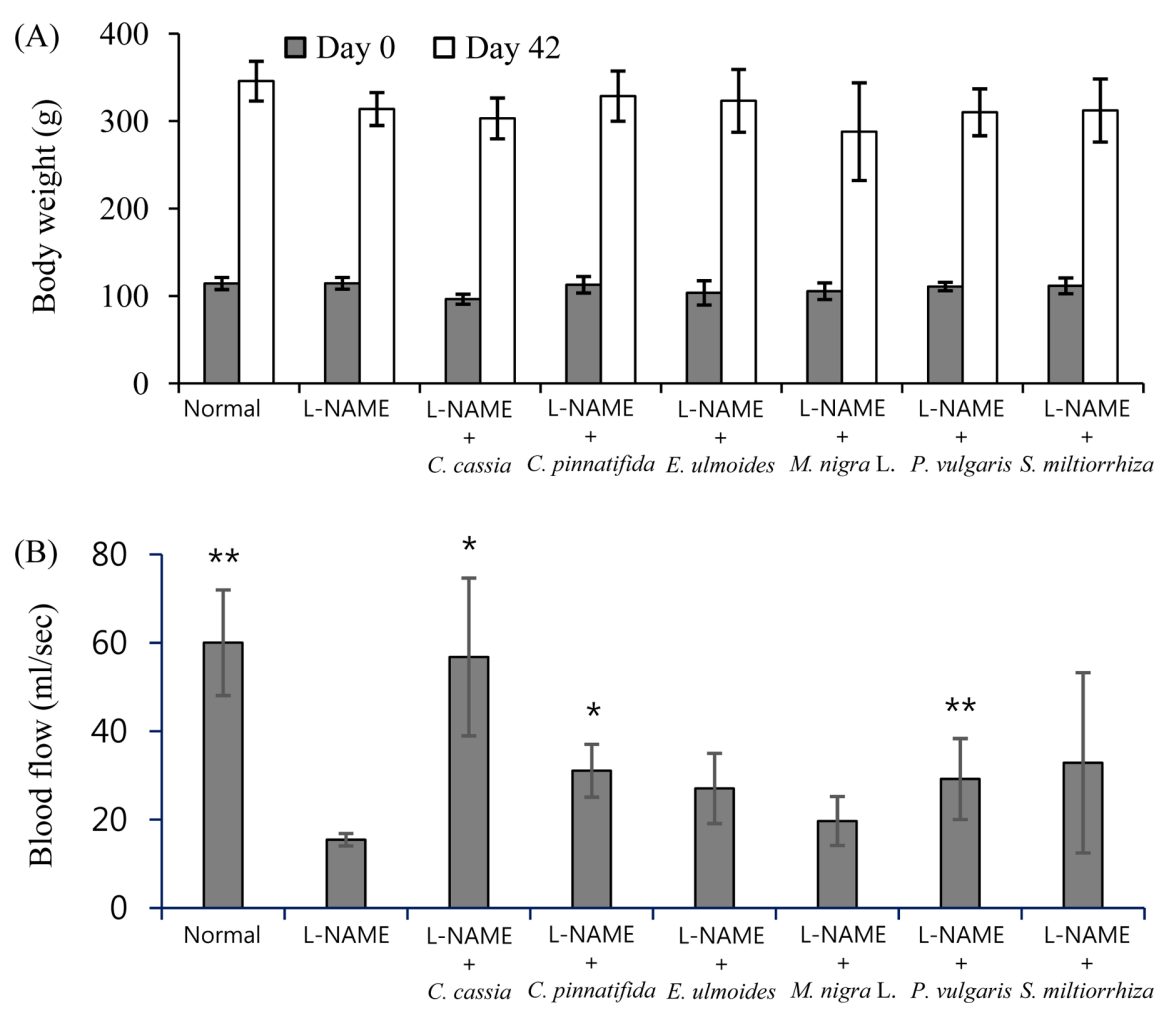

Fig. 1. Effect of plants extracts on body weight and blood flow of L.NAME induced arthrosclerosis rats. (A) The body weights of the animals were measured on day 0 and 42. (B) Blood flows were measured at the date of sacrifices. The data from three independent experiments, each of which was performed in triplicate, are indicated as mean $\pm \mathrm{SD}$. Statistical significance $\left({ }^{*} p<0.05 ;{ }^{* *} p<0.01\right.$, Student's t-test $)$ as compared with L-NAME model

Effect of $C$. cassia and $S$. miltiorrhiza extracts on blood flow To confirm the positive effects of $C$. cassia and S. miltiorrhiza extracts on blood flow, the extracts were administered to rats, either individually or in combination. As shown in Fig. 2(B), blood flow was reduced by L-NAME treatment, but this deleterious effect was ameliorated by treatment with C. cassia and S. miltiorrhiza extracts, either singly or in combination. This finding indicates that the plant extracts cause vasodilation of the carotid artery.

Effect of $C$. cassia and $S$. miltiorrhiza extracts on the blood vessels of L-NAME-treated animals

To further determine the effects of $C$. cassia and S. miltiorrhiza extracts on blood vessels, we analyzed the wall thickness of the ascending aorta histopathologically. Ascending aortas were prepared as $5 \mu \mathrm{m}$-thick sections and then stained with H\&E. As shown in Fig. 3, the ascending aortae of L-NAME-treated rats had almost three-fold thicker walls than those of control rats, indicating hypertrophy. However, the thickness of the aorta walls was significantly reduced by treatment with $C$. cassia or S. miltiorrhiza extracts. Rats treated with extracts of a single plant had aortic walls that were still two-fold thicker than those of the control group, but treatment with a mixture of C. cassia and S. miltiorrhiza extracts had a larger effect, which was not dose-dependent. Other vascular histologic changes, like as connective tissue layer of adventitia, were also observed in the L-NAME-treated rats, which

Table 1 Changes of blood pressure in L-NAME treated rats

\begin{tabular}{|c|c|c|c|c|c|c|c|}
\hline Variables & Normal & L-NAME & $\begin{array}{c}\text { L-NAME } \\
+ \\
\text { C. cassia } \\
150 \mathrm{mg} / \mathrm{kgbw}\end{array}$ & $\begin{array}{c}\text { L-NAME } \\
+ \\
\text { S. miltiorrhiza } \\
150 \mathrm{mg} / \mathrm{kgbw}\end{array}$ & $\begin{array}{c}\text { L-NAME } \\
+ \\
\text { Mixture } \\
150 \mathrm{mg} / \mathrm{kgbw}\end{array}$ & $\begin{array}{c}\text { L-NAME } \\
+ \\
\text { Mixture } \\
300 \mathrm{mg} / \mathrm{kgbw}\end{array}$ & $\begin{array}{c}\text { L-NAME } \\
+ \\
\text { Mixture } \\
600 \mathrm{mg} / \mathrm{kgbw}\end{array}$ \\
\hline SBP & $92 \pm 5.68$ & $164 \pm 4.98$ & $158 \pm 7.35$ & $151 \pm 6.98$ & $148 \pm 8.15$ & $131 \pm 3.54^{*}$ & $124 \pm 7.39^{*}$ \\
\hline DBP & $65 \pm 8.64$ & $132 \pm 8.23$ & $129 \pm 3.81$ & $128 \pm 6.81$ & $115 \pm 2.52 *$ & $118 \pm 4.82 *$ & $92 \pm 6.15^{*}$ \\
\hline MAP & $89 \pm 3.23$ & $152 \pm 6.12$ & $147 \pm 2.82$ & $148 \pm 5.37$ & $137 \pm 2.92 *$ & $129 \pm 8.17 *$ & $113 \pm 7.13^{*}$ \\
\hline
\end{tabular}

Data are presented as mean $\pm \mathrm{SD},(\mathrm{n}=5, p<0.05)$. Statistical significance $\left({ }^{*} p<0.05\right.$, Student's t-test $)$ as compared with L-NAME group. Systolic Blood Pressure (SBP); Diastolic Blood Pressure (DBP); Mean Arterial Pressure (MAP) 

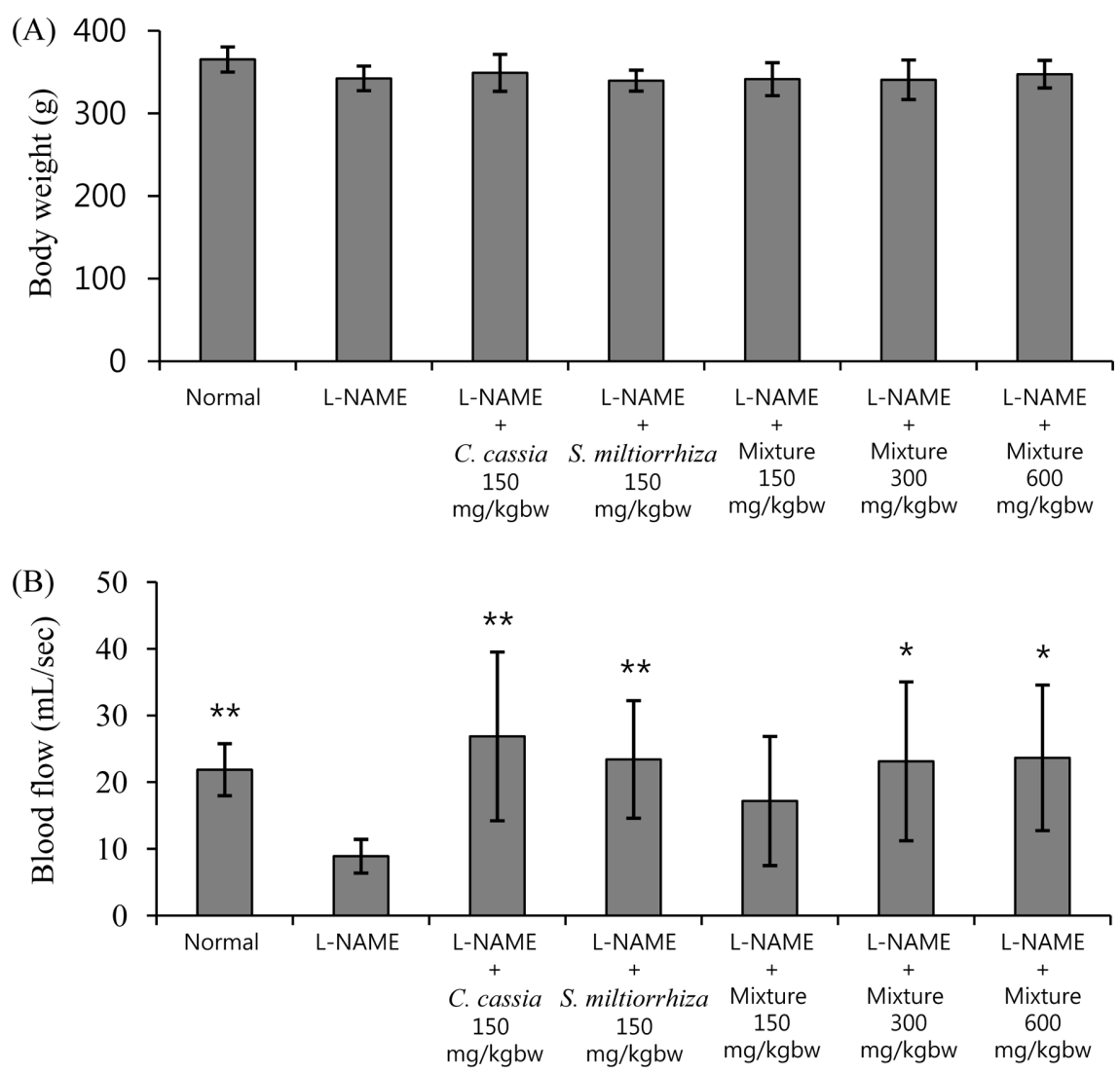

Fig. 2. Effect of C. cassia and S. miltiorrhiza extracts on body weight and blood flow of L-NAME model rats. The body weights (A) and blood flows (B) of the animals were measured on the day of sacrifice. The data from three independent experiments, each of which was performed in triplicate, are indicated as mean $\pm \mathrm{SD}$. Statistical significance $\left({ }^{*} p<0.05 ;{ }^{* *} p<0.01\right.$, Student's t-test $)$ as compared with L-NAME model

were ameliorated in rats treated with plant extracts (Fig. 3).

\section{Effect of $C$. cassia and $S$. miltiorrhiza on eNOS protein expression}

L-NAME is an inhibitor of NOS activity, both in vivo and in vitro; therefore, L-NAME induces hypertension and damage in vascular tissues because of a reduction in NO synthesis [6,7]. The eNOS protein expression was confirmed by western blotting of liver tissue lysates. The eNOS expression in liver lysates was significantly lower in L-NAME-treated rats than in control rats, but the plant extracts significantly ameliorated this reduction in eNOS, except for the $150 \mathrm{mg} / \mathrm{kgbw}$ mixture (Fig. 4). The highest eNOS expression levels were found in rats that were administered the combination of plant extracts at $600 \mathrm{mg} / \mathrm{kgbw}$.

\section{Intracellular ROS production}

ROS provokes an inflammatory reaction by inducing oxidative damage in vascular endothelial cells and ultimately induces vascular wall dysfunction. To determine whether ROS production is inhibited by the plant extracts, oxidative stress was induced in vascular endothelial cells (HUVEC cells) by treatment with $\mathrm{H}_{2} \mathrm{O}_{2}$, and the cells were then treated with the $C$. cassia and S. miltiorrhiza extracts. As shown in Fig. $5 \mathrm{~A}, \mathrm{H}_{2} \mathrm{O}_{2}$ treatment significantly increased ROS production, but this effect was ameliorated by treatment with either single extracts or mixtures of $C$. cassia and $S$. miltiorrhiza. The largest reduction in ROS production was induced by the highest concentration of extracts in a 1:1 mixture of $C$. cassia and S. miltiorrhiza extracts.

\section{Nitric oxide release}

To further clarify the anti-oxidative action of the plant extract mixture, we compared the amounts of NO produced by HUVEC cells in vitro after each treatment. NO mediates blood vessel relaxation and permeability; therefore, we measured $\mathrm{NO}$ production after inducing oxidative stress by treatment with $\mathrm{H}_{2} \mathrm{O}_{2}$. As shown in Fig. 5B, the cells' release of $\mathrm{NO}$ was reduced by treatment with $\mathrm{H}_{2} \mathrm{O}_{2}$, but this reduction was limited by treatment with a mixture of $C$. cassia and S. miltiorrhiza extracts.

\section{Effect of $C$. cassia and $S$. miltiorrhiza on the production of} proinflammatory cytokines

Damage to blood vessels induces inflammation, which involves inflammatory cells, including macrophages and dendritic cells. Activated macrophages secrete pro-inflammatory cytokines, such 


\begin{tabular}{|c|c|c|c|c|c|c|c|}
\hline & Normal & L-NAME & $\begin{array}{c}\text { L-NAME } \\
+ \\
\text { C. cassia } \\
150 \mathrm{mg} / \mathrm{kgbw}\end{array}$ & $\begin{array}{c}\text { L-NAME } \\
+ \\
\text { S. } \text { miltiorrhiza } \\
150 \mathrm{mg} / \mathrm{kgbw}\end{array}$ & $\begin{array}{c}\text { L-NAME } \\
+ \\
\text { Mixture } \\
150 \mathrm{mg} / \mathrm{kgbw}\end{array}$ & $\begin{array}{c}\text { L-NAME } \\
+ \\
\text { Mixture } \\
300 \mathrm{mg} / \mathrm{kgbw}\end{array}$ & $\begin{array}{c}\text { L-NAME } \\
+ \\
\text { Mixture } \\
600 \mathrm{mg} / \mathrm{kgbw}\end{array}$ \\
\hline \multicolumn{8}{|l|}{ Cross section } \\
\hline \multicolumn{8}{|c|}{ Wall thickness } \\
\hline Thickness & $\begin{array}{c}125.42 \\
\pm 5.66 \mu \mathrm{m}\end{array}$ & $\begin{array}{c}378.07 \\
\pm 13.12 \mu \mathrm{m}\end{array}$ & $\begin{array}{c}267.56 \\
\pm 11.59 \mu \mathrm{m}\end{array}$ & $\begin{array}{c}271.74 \\
\pm 17.62 \mu \mathrm{m}\end{array}$ & $\begin{array}{c}154.70 \\
\pm 11.65 \mu \mathrm{m}\end{array}$ & $\begin{array}{c}148.94 \\
\pm 18.15 \mu \mathrm{m}\end{array}$ & $\begin{array}{c}158.33 \\
\pm 15.79 \mu \mathrm{m}\end{array}$ \\
\hline
\end{tabular}

Fig. 3. Histopathological analysis of ascending aorta. The tissues of ascending aorta were cut as $5 \mu \mathrm{m}$ thickness and then stained with H\&E staining. Upper panel, magnification of $\times 50$ (cross section); lower panel, magnification of $\times 400$ (wall thickness). The data shown are representative of three independent experiments

as tumor necrosis factor (TNF)- $\alpha$, interleukin (IL)-1 $\beta$, and IL-6. These cytokines are important initiators of the inflammatory response and play a role in developing a variety of inflammatory diseases [16]. Therefore, the murine macrophage cell line, Raw 264.7, was pretreated with the plant extracts and then stimulated with lipopolysaccharide (LPS). As shown in Fig. 6, TNF- $\alpha$, IL-6, and IL-1 $\beta$ production was significantly increased following $1 \mu \mathrm{g} /$ $\mathrm{mL}$ LPS stimulation, but their concentrations were reduced by pretreatment with $C$. cassia and S. miltiorrhiza extracts. The mixture of $C$. cassia and $S$. miltiorrhiza extracts reduced TNF- $\alpha$ and IL-6 production, which may be beneficial for the vasodilatory and antihypertensive properties of the artery.

\section{Discussion}

Although there are still few effective treatments for hypertension, there are several types of natural plant extracts that have antihypertensive effects [14]. In this study, we have used L-NAME to induce vascular disease in rats. This model has provided significant information regarding the etiology, pathogenesis, and sequelae of such disease, and also provides a means for testing the efficacy of candidate therapeutic compounds. This model is also useful as a model of atherosclerosis, because the reduction in NO production induced by L-NAME results in hyperlipidemia [17]. In the present study, we used this model to screen six plant extracts for their antiatherosclerotic and anti-hypertensive effects, and then selected extracts of $C$. cassia and $S$. miltiorrhiza for further study.

C. cassia and S. miltiorrhiza are commonly used as Chinese herbal medicines to treat cardiovascular diseases. They have been shown to have pharmaceutical effects in the cardiovascular system: anti-thrombotic effects, cardioprotective effects, anti-hypertensive effects, and anti-atherosclerotic effects. They also maintain the microcirculation in the brain and heart $[18,19]$. Both extracts have been shown to promote blood flow and improve the circulation in the heart $[18,19]$. Improvements in blood flow were also shown using laser Doppler blood flowmetry in vivo in the present study. L-NAME-treated rats had lower blood flow $(8.9 \mathrm{~mL} / \mathrm{sec})$ than control rats, but when treated with $C$. cassia $(26.87 \mathrm{~mL} / \mathrm{sec})$ and S. miltiorrhiza $(23.42 \mathrm{~mL} / \mathrm{sec})$ alone, or with a combination at low $(17.18 \mathrm{~mL} / \mathrm{sec})$, intermediate $(23.13 \mathrm{~mL} / \mathrm{sec})$, or high $(23.65 \mathrm{~mL} /$ $\mathrm{sec})$ concentrations, there were substantial improvements in blood flow, which returned it to near normal $(21.87 \mathrm{~mL} / \mathrm{sec})$.

According to a previous report, vascular fibrosis, which is characterized by lumen diameter reduction and thickening of the coronary artery wall, can be attributed to degradation of the adventitia and inhibition of matrix degradation [19]. The analysis of arterial tissue in a vascular disease model is typically performed by microscopic examination of the intima, media, and adventitia [20]. In the present study, aortic vessel layer remodeling was classified in all six experimental groups. The control group was characterized by a clear separation of the three layers, whereas LNAME-treated rat aortae showed significant degradation of the intima and adventitia. However, remodeling of the intima (in which endothelial cells are in direct contact with the blood) and adventitia was observed in all the treatment groups. Furthermore, the aortae of L-NAME-treated rats were thicker in cross-section than those of control rats, indicating hypertrophy. However, the thickness was significantly reduced in rats treated with $C$. cassia and $S$. miltiorrhiza extracts.

In the present study, the plant extracts ameliorated the LNAME-induced hypertrophy of the aortic wall, even at the lowest doses, which was likely mediated by an increased nitric oxide synthase expression. It is known that eNOS expression is an 


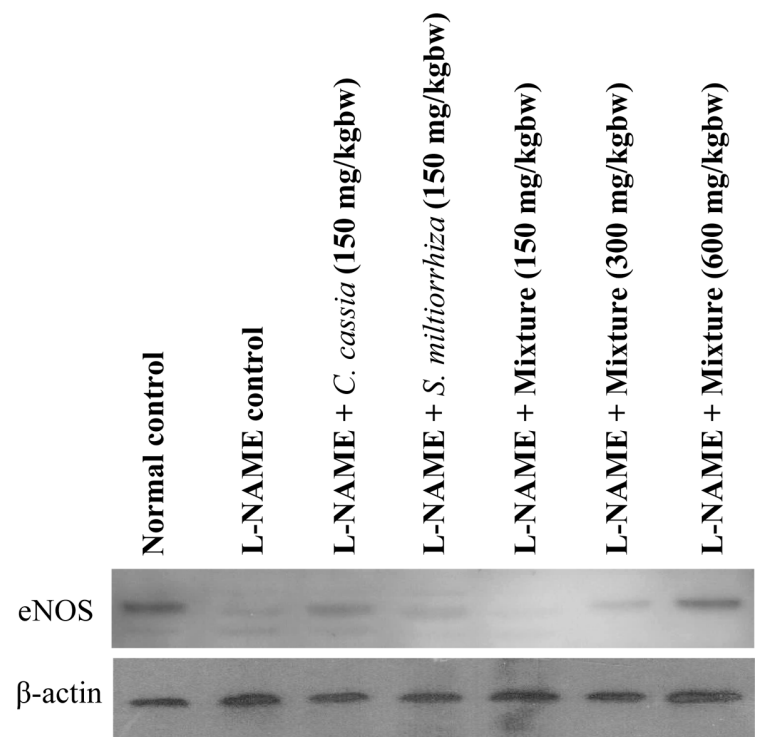

Fig. 4. Effect of C. cassia and $S$. miltiorrhiza extracts on eNOS protein expression. The expression of eNOS was confirmed by western blot with the extract of liver tissue. The data shown are representative of three independent experiments

important mediator of physiologic vasodilatation in rats, and in our in vivo study, L-NAME reduced both blood flow and the expression of eNOS, both of which were restored by treatment with $C$. cassia or $S$. miltiorrhiza extracts. This is consistent with an indirect mechanism, depending on $\mathrm{NO}$ synthesis and release from endothelial cells, mediating blood vessel relaxation [21]. We have also demonstrated that $C$. cassia and $S$. miltiorrhiza extracts increase NO production by endothelial cells in vitro.

C. cassia and S. miltiorrhiza extracts mitigated ROS production by oxidatively damaged HUVEC cells. ROS play a role as second messengers in many immune responses [22] and as cytolytic molecules involved in killing pathogens [23, 24]. Oxidative stress is considered a key component of the pathogenesis of atherosclerosis [25]. The production of large amounts of ROS leads to vasoconstriction, vascular remodeling, inflammation, and fibrosis [26]. An inflammatory reaction occurs secondary to oxidative damage to vascular endothelial cells and ultimately induces dysfunction of the vascular wall.

Numerous studies have shown that oxidative stress leads to production of proinflammatory cytokines [27], thereby linking ROS with inflammation and vascular endothelial dysfunction. Inflammation is the immune system's response to infection, injury, or irritation, and involves the production of pro-inflammatory mediators, which increase or suppress the production of other inflammatory mediators, such as NO, cyclooxygenase, and prostaglandins [28]. Macrophages are the most common cell type used to screen potential anti-inflammatory compounds because they are at the center of the inflammatory response and initiate the secretion of a cytokine cascade. Activated macrophages secrete proinflammatory cytokines, such as TNF- $\alpha$, IL-1 $\beta$, and IL-6,
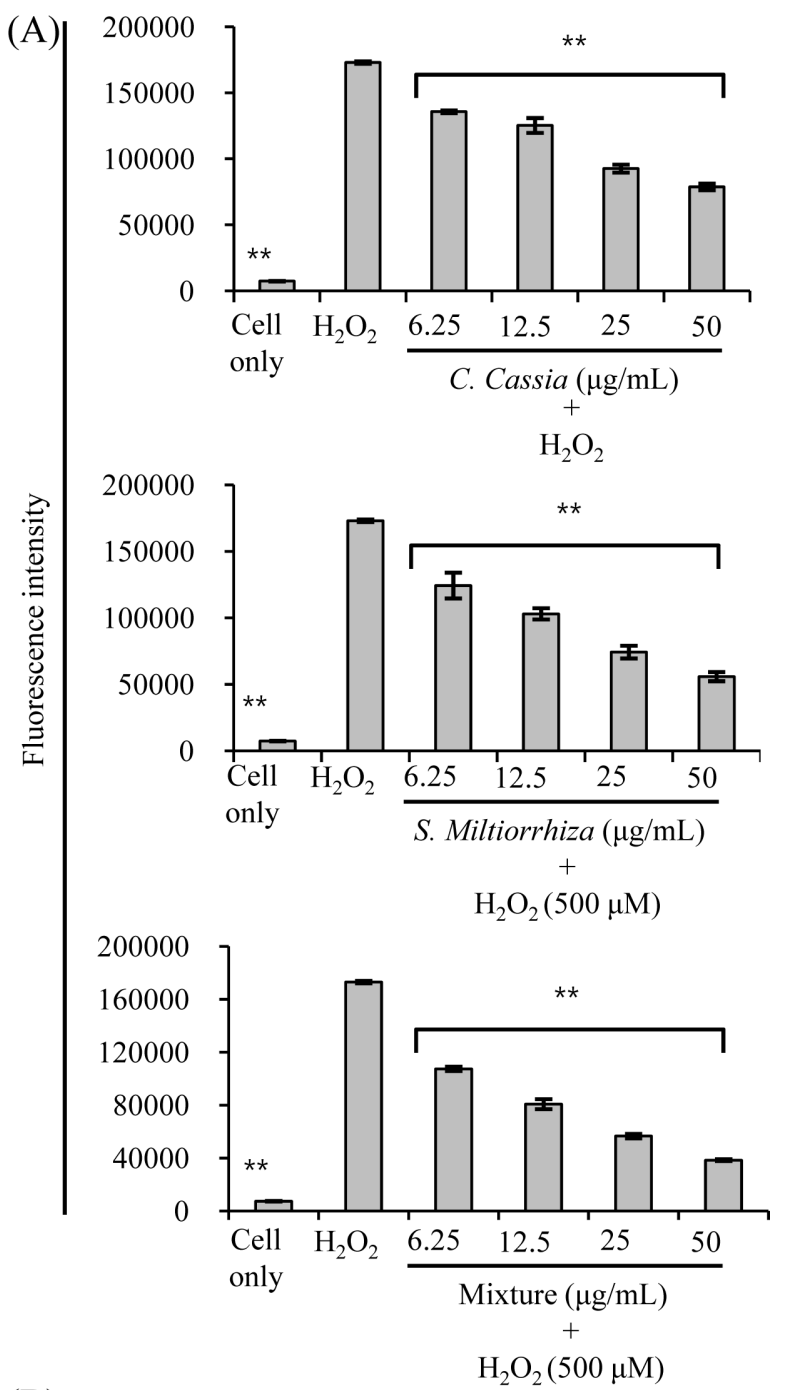

(B)

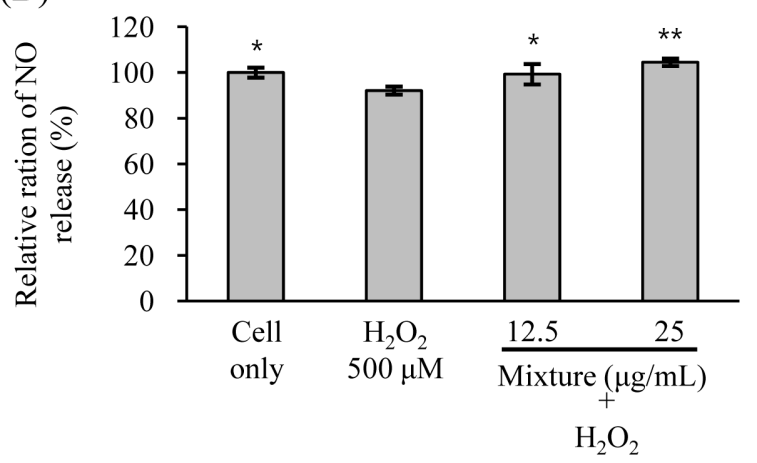

Fig. 5. Effect of C. cassia and S. miltiorrhiza extracts on ROS (A) and NO (B) production from HUVEC cells. The data from three independent experiments, each of which was performed in triplicate, are indicated as mean \pm SD. Statistical significance $(* * p<0.01$, Student's t-test $)$ as compared with $\mathrm{H}_{2} \mathrm{O}_{2}$ control

which are essential for the inflammatory response and are involved in many inflammatory diseases [29]. Anti-inflammatory effects $C$. cassia and $S$. miltiorrhiza extracts have already been 

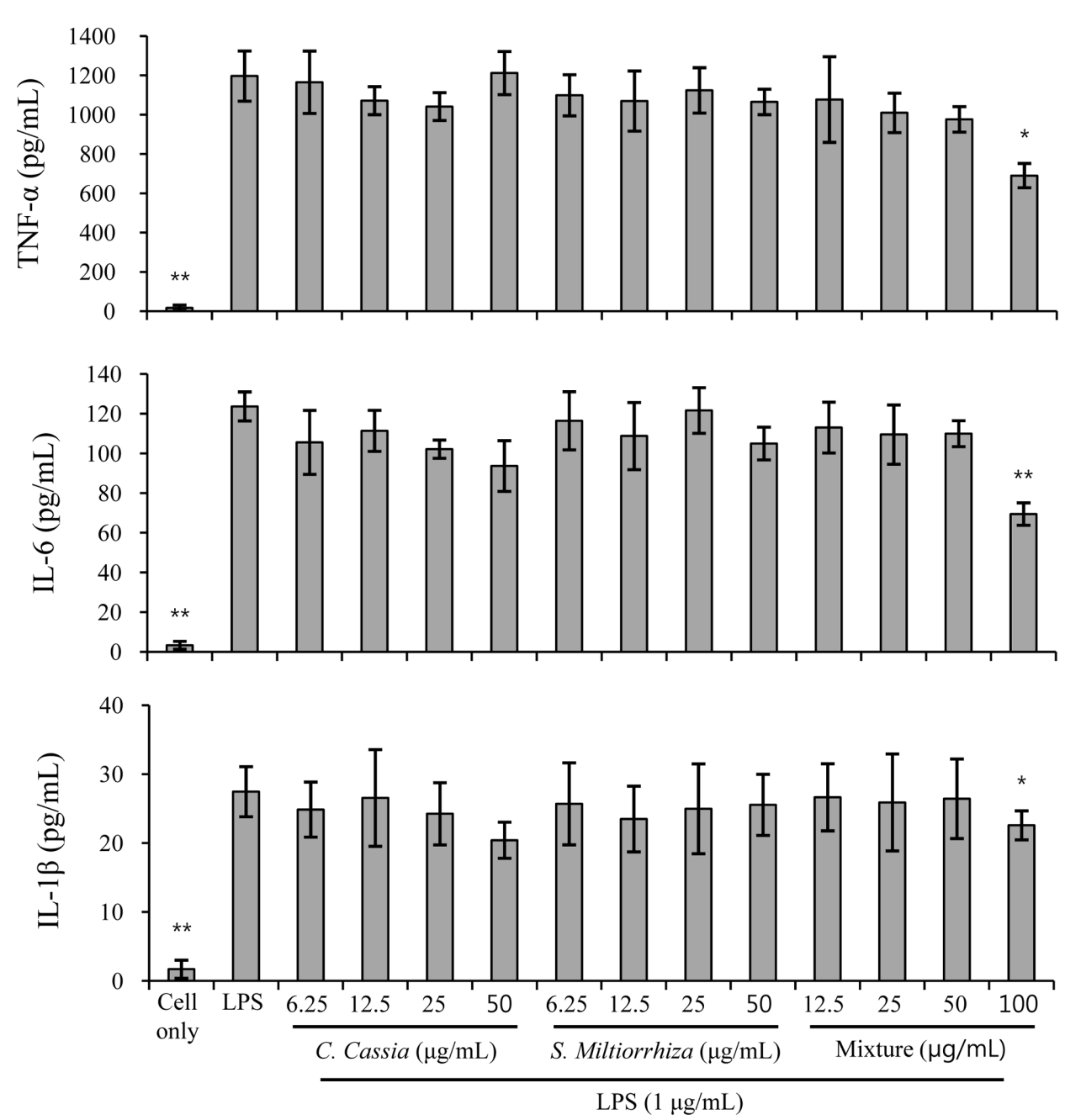

Fig. 6. Effect of C. cassia and S. miltiorrhiza on the production of proinflammatory cytokines. Proinflammatory cytokines were measured by ELISA assay after sampling the culture supernatants. The data from three independent experiments, each of which was performed in triplicate, are indicated as mean \pm SD. Statistical significance $\left({ }^{*} p<0.05 ;{ }^{*} p<0.01\right.$, Student's t-test) as compared with LPS control

demonstrated in the treatment of cardiovascular diseases [29]. In the present study, we have also identified anti-inflammatory properties of ethanolic plant extracts of $C$. cassia and $S$. miltiorrhiza, alone and mixed, because they suppressed TNF- $\alpha$, IL-1 $\beta$, and IL6 production by LPS-stimulated macrophages. The antiinflammatory properties of $C$. cassia and S. miltiorrhiza shown in the in vitro experiment appeared to be similar to those of other compounds, such as cinnamic aldehyde [29], which can be derived from $C$. cassia. We also attempted to demonstrate the effects of the extracts on TNF- $\alpha$, IL- $1 \beta$, and IL- 6 production in vivo, but the cytokine concentrations were too low to measure.

In this study, we have demonstrated additive effects of a mixture of $C$. cassia and $S$. miltiorrhiza extracts in L-NAME-treated hypertensive rats. This mixture causes a moderate reduction in blood pressure, and ameliorates reduced blood flow and abnormal aortic histology induced by L-NAME. Although further studies are required to identify the mechanism involved, we present evidence that the anti-hypertensive and anti-atherosclerotic effects of this mixture may be caused by upregulation of eNOS expression, and anti-oxidative and anti-inflammatory activities.

\section{References}

1. O'Flaherty M, Flores-Mateo G, Nnoaham K, Lloyd-Williams F, Capewell S (2102) Potential cardiovascular mortality reductions with stricter food policies in the United Kingdom of Great Britain and Northern Ireland. Bull World Health Organ 90 (7): 522-531

2. Oparil S, Czarina Acelajado M, Bakris GL, Dan R. Berlowitz, Cífková R, Guido Grassi AF, JordanJ, Poulter NR, Rodgers A, Whelton PK (2008) Hypertension. Nat Rev Dis Primers 4: 18014

3. Liu WT, Huang PL (2008) Cardiovascular roles of nitric oxide: A review of insights from nitric oxide synthase gene disrupted mice. Cardiovasc Res 77 (1): 19-29

4. Wu J, Xiz S, Kalionis B, Wan W, Sun T (2014) The Role of Oxidative Stress and Inflammation in Cardiovascular Aging. BioMed Research 
International 2014: 1-13

5. Berkban T, Boonprom P, Bunbupha S, Umka Welbat J, Kukongviriyapan U, Kukongviriyapan V, Pakdeechote P, Prachaney P (2015) Ellagic Acid Prevents L-NAME-Induced Hypertension via Restoration of eNOS and p47phox Expression in Rats. Nutrients 7: 5265-5280

6. Nakmareong S, Kukongviriyapan U, Pakdeechote P, Donpunha W, Kukongviriyapan V, Kongyingyoes B, Sompamit K, Phisalaphong C (2011) Antioxidant and vascular protective effects of curcumin and tetrahydrocurcumin in rats with L-NAME-induced hypertension. Naunyn Schmiedebergs Arch Pharmacol 383: 519-529

7. Sung JH, Jo YS, Kim SJ, Ryu JS, Kim MC, Ko HJ, Sim SS (2013) Effect of Lutein on L-NAME-Induced Hypertensive Rats. Korean J Physiol Pharmacol 17: 339-345

8. Klima L, Kawecka-Jaszcz K, Stolarz-Skrzypek K, Menne, J.; Fijorek, K.; Olszanecka, A.; Wojciechowska, W.; Bilo, G.; Czarnecka, D (2013) Structure and function of large arteries in hypertension in relation to oxidative stress markers. Kardiol Pol 71: 917-923

9. Jiaur R. Gayen, Kuixing Zhang, Satish P. RamachandraRao, Manjula Mahata, Yuqing Chen, Hyung-Suk Kim, Robert K. Naviaux, Kumar Sharma, Sushil K. Mahata, Daniel T. O'Connor (2010) Role of Reactive Oxygen Species in Hyper-Adrenergic Hypertension: Biochemical, Physiological, and Pharmacological Evidence from Targeted Ablation of the Chromogranin A (Chga) Gene. Circ Cardiovasc Genet 3 (5): 414 425

10. Litterio, MC, Jaggers, G, Sagdicoglu Celep G, Adamo AM, Costa MA, Oteiza PI, Fraga CG, Galleano M (2012) Blood pressure-lowering effect of dietary ()-epicatechin administration in L-NAME-treated rats is associated with restored nitric oxide levels. Free Radic Biol Med 53: 1894-1902

11. Schulte W, Bernhagen J, Bucala R (2013) Cytokines in Sepsis: Potent Immunoregulators and Potential Therapeutic Targets-An Updated View. Mediators Inflamm 6: 165974

12. Mittal M, Rizwan Siddiqui M, Tran K, Reddy SP, Malik AB (2014) Reactive Oxygen Species in Inflammation and Tissue Injury. Antioxid Redox Signal. 20(7): 1126-1167

13. Okwuonu CG, Ojimadu NE, Okaka EI, Akemokwe FM (2014) Patientrelated barriers to hypertension control in a Nigerian population. Int $\mathrm{J}$ Gen Med 7: 345-353

14. Liu C, Huang Y (2016) Chinese Herbal Medicine on Cardiovascular Diseases and the Mechanisms of Action. Front Pharmacol 7: 469

15. Yang C-H, Li R-X, Chuang L-Y (2012) Antioxidant Activity of Various Parts of Cinnamomum cassia Extracted with Different Extraction Methods. Molecules 17: 7294-7304

16. Brennan FM, Maini RN, Feldmann M (1998) Role of pro-inflammatory cytokines in rheumatoid arthritis. Springer Semin Immunopathol. 20: 133-147
17. Aluko EO, Omobowale TO, Oyagbemi AA, Adejumobi OA, Ajibade TO, Fasanmade AA (2018) Reduction in nitric oxide bioavailability shifts serum lipid content towards atherogenic lipoprotein in rats. Biomed Pharmacother 101: 792-797

18. Liao JC, Deng J-S, Chiu C-S, Hou W-C, Huang S-S, Shie P-H, Huang G-J (2012) Anti-Inflammatory Activities of Cinnamomum cassia Constituents In Vitro and In Vivo. Evid Based Complement Alternat Med 11: 429320

19. He ZD, Qiao CF, Han QB, Cheng CL, Xu HX, Jiang RW, But PP, Shaw PC (2005) Authentication and quantitative analysis on the chemical profile of Cassia Bark (Cortex Cinnamomi) by high-pressure liquid chromatography. J Agr Food Chem 53 (7): 2424-2428

20. Lan TH, Huang XQ, Tan HM (2013) Vascular fibrosis in atherosclerosis. Cardiovasc Pathol 22(5): 401-407

21. Li CM, Dong XL, Fan XD, Wu JH, Wang QH, Tian XL, Guo DJ, Wong MS, Qiu TQ, Chan SW (2013) Aqueous extract of danshen (Salvia miltiorrhiza Bunge) protects ovariectomized rats fed with high-fat diet from endothelial dysfunction. Menopause 20(1): 100-109

22. Mittal M, Siddiqui MR, Tran K, Reddy SP, Malik AB (2014) Reactive oxygen species in inflammation and tissue injury. Antioxid Redox Signal 20 (7): 1126-1167

23. Nishikawa T, Edelstein D, Du XL, Yamagishi S, Matsumura T, Kaneda Y, Yorek MA, Beebe D, Oates PJ, Hammes HP, Giardino I, Brownlee M (2000) Normalizing mitochondrial superoxide production blocks three pathways of hyperglycaemic damage. Nature 404 (6779): 787-790

24. Schleicher E, Friess U (2007) Oxidative stress, AGE, and atherosclerosis. Kidney Int 72 (Suppl. 106): S17-S26

25. Rodriguez-Porcel M, Chade AR, Miller JD (2017) Studies on Atherosclerosis. Oxidative Stress in Applied Basic Research and Clinical Practice, 1st Edn. Berlin: Springer. 10.1007/978-1-4899-7693-2

26. Bulua AC, Simon A, Maddipati R, Pelletier M, Park H, Kim KY, Sack MN, Kastner DL, Siegel RM (2011) Mitochondrial reactive oxygen species promote production of proinflammatory cytokines and are elevated in TNFR1-associated periodic syndrome (TRAPS). J Exp Med 208(3): 519-533

27. Chen L, Deng H, Cui H, Fang J, Zuo Z, Deng J, Li Y, Wang X, Zhao L (2018) Inflammatory responses and inflammation-associated diseases in organs. Oncotarget 9(6): 7204-7218

28. Johnson GL, Lapadat R (2002) Mitogen-activated protein kinase pathways mediated by ERK, JNK, and p38 protein kinases. Science 298(5600): 1911-1912

29. Lin SS, Lu TM, Chao PC, Lai YY, Tsai HT, Chen CS, Lee YP, Chen SC, Chou MC, Yang CC (2011) In vivo cytokine modulatory effects of cinnamaldehyde, the major constituent of leaf essential oil from Cinnamomum osmophloeum Kaneh. Phytother Res 25(10): 1511-1518 